\title{
Amorphization-assisted nanoscale wear during the running-in process
}

\author{
Xiaoli $\mathrm{Hu}^{\mathrm{a}}$, M. Virginia P. Altoe ${ }^{\mathrm{b}}$, Ashlie Martini ${ }^{\mathrm{a}, *}$ \\ ${ }^{a}$ School of Engineering, University of California, Merced, CA 95343 \\ ${ }^{b}$ Molecular Foundry, Lawrence Berkeley National Laboratory, Berkeley, CA 94720
}

\begin{abstract}
Atomistic simulations were used to study the nanoscale wear of crystalline silicon with a native oxide sliding against amorphous silicon dioxide. The size, shape and crystallographic orientation of the model were defined to be comparable to those in a corresponding atomic force microscope experiment, where the tip was imaged before and after $40 \mathrm{~nm}$ of sliding using ex situ transmission electron microscopy. Tip wear was quantified in the simulation as the volume of silicon atoms removed from the tip at intervals up to $40 \mathrm{~nm}$ sliding distance. We also quantified amorphization during sliding as the change of tip material from crystalline to amorphous. The rate of amorphization decreased with sliding distance, and this trend was analyzed in the context of a previously-proposed analytical model for crystalline-to-amorphous transitions and explained qualitatively by local strain distributions within the tip. Both wear and amorphization were found to exhibit similar trends, but the wear rate was faster than the rate of amorphization. The results suggest that amorphization may be a dominant mechanism of nanoscale wear during the early stages of running-in, but less so during steady-state sliding.

Keywords: Molecular dynamics, Nanoscale wear, Amorphization, Running-in wear
\end{abstract}

\footnotetext{
*Corresponding author. Tel.: +1 2092282354 .

Email address: amartini@ucmerced.edu (Ashlie Martini)
}

Preprint submitted to Wear

September 5, 2016

(C) 2016. This manuscript version is made available under the Elsevier user license http://www.elsevier.com/open-access/userlicense/1.0/ 


\section{Introduction}

Since its invention, atomic force microscopy (AFM) has been widely used for studying surface features and manipulating materials from the atomic to the micron scale. The quality of AFM-based measurements and manufacturing processes are critically dependent on the reliability and durability of AFM tip itself [1-5]. However, the contact between the tip and sample, and the associated high mechanical stress and/or chemical reactions, can result in wear of the tip during use [6-13]. Tip wear is undesirable because it can result in an inability to resolve fine structures during AFM implementation [14]. These wear processes are necessarily nanoscale, suggesting that standard, macroscale wear theories may not always be applicable to describe or predict tip wear. Thus, a fundamental understanding of wear mechanisms at the nanoscale is a critical first step towards ensuring the structural and chemical integrity of an AFM tip during instrument use.

Recently, a number of experimental studies have used AFM to characterize nanoscale wear on a variety of different material systems $[6,7,9,15]$. These studies have been complemented by molecular dynamics (MD) simulation of the AFM tip apex sliding on well-defined substrate surfaces $[12,13,16]$. One observation from such studies is that a crystalline-to-amorphous transition may contribute to nanoscale wear. Experimental measurements of sliding on nanocrystalline diamond revealed that amorphous carbon was formed by the wear process $[17,18]$. The observation was explained theoretically by an MD study of a diamond-diamond interface that showed mechanical amorphization drives wear [19]. These studies suggest that wear at the nanoscale may not be a simple process of material removal, but rather may be assisted by material change during sliding. Further complicating the issue is that wear rate is not only a materialand condition-specific property, it is also dependent on how long sliding has progressed. At the onset of sliding, the system is "running-in" and the wear rate can be orders of magnitude higher than that during steady state wear [9]. During running-in, the two sliding partners adjust each other in terms of stress, 
surface roughness or crystal orientations [20-22]. The chemical and mechanical processes that occur during running-in are expected to play an important role in steady state wear [20]. This means that understanding the processes that lead to material removal in the first tens of nanometers of sliding is critical to understanding nanoscale wear in general.

In this work, MD simulations were used to study the nanoscale wear of a crystalline silicon AFM tip with a native oxide due to sliding against an amorphous silicon dioxide substrate. The size, shape and crystallographic orientation of the tip apex in the MD model were defined to be comparable to those in a corresponding AFM experiment, where the tip was imaged before and after 40 nm of sliding using ex situ transmission electron microscopy (TEM). Tip wear was quantified in the simulation as the volume of silicon atoms removed from the tip at intervals up to the same $40 \mathrm{~nm}$ sliding distance as in the experiment. We also quantified tip amorphization as the change of amorphous tip volume due to sliding. The amorphization process was analyzed in the context of a previously-proposed model for sliding-induced amorphization and explained in terms of local strain within the tip. Lastly, the wear and amorphization rates were evaluated together to determine how these two processes are related and determine the running-in process at the nanoscale.

\section{Methods}

The simulation was designed to mimic an AFM experiment in which a silicon tip was slid against a freshly-annealed amorphous $\mathrm{SiO}_{2}$ substrate $\left(1050^{\circ} \mathrm{C}, 15\right.$ $\mathrm{h}$ in air) under dry nitrogen (0.026 bar above ambient) and stabilized temperature $(28 \pm 5)^{\circ} \mathrm{C}$. In the experiment, non-contact AFM images and force distance curves (maximum load of $5 \mathrm{nN}$ ) were obtained immediately before sliding. The tip was brought into contact with the surface maintaining the silicon crystallographic direction $\left[\begin{array}{lll}1 & 1 & 7\end{array}\right]$ perpendicular to the sample surface and loaded to $100 \mathrm{nN}$. Then the tip was slid, in contact with the surface, along the $\left[\begin{array}{lll}1 & -1 & 0\end{array}\right]$ silicon crystallographic direction at constant normal load of $100 \mathrm{nN}$ and speed of 
$2.5 \mu \mathrm{m} / \mathrm{s}$. The tip was imaged using high resolution TEM (HRTEM) before and after sliding $40 \mathrm{~nm}$. Experimental tip wear volume was calculated by overlaying the HRTEM images obtained before and after wear experiment, measuring the difference in area of those $2 \mathrm{D}$ projections and assuming tip was circularly symmetric in the third dimension (truncated cone approximation).

Consistent with the materials in experiments, we modeled a Si tip with an oxidized surface and an amorphous $\mathrm{SiO}_{2}$ substrate. From the high-resolution tip image obtained from TEM before sliding, shown in Figure 1(a), the tip profile was extracted and was fit with a parabola, indicated by a dashed red line in the figure. Based on this profile, a corresponding parabolic tip was cut from a cubic model of crystalline silicon. The radius of curvature of the tip profile was estimated to be $1 \mathrm{~nm}$, and the model tip height of $10 \mathrm{~nm}$ was sufficient to capture the wear mechanisms expected under the conditions explored here. Then the tip was placed in an atmosphere of atomic oxygen. After the reaction between silicon atoms and oxygen atoms, unbonded oxygen atoms were removed from the simulation box. A cross section of the oxygen terminated tip is shown in Figure 1(b). The amorphous $\mathrm{SiO}_{2}$ substrate was created using Visual Molecular Dynamics (VMD) [23, 24]. The dimensions of the substrate were $23.2 \times 11.4 \times$ $3.5 \mathrm{~nm}$ in the $x^{-}, y-$, and $z$-directions, respectively. To maintain computation efficiency, we did not initially set the substrate long enough to accommodate the target sliding distance, i.e. $40 \mathrm{~nm}$. However, when the tip was about to exceed the boundary during sliding, we removed part of worn substrate and added a new amorphous $\mathrm{SiO}_{2}$ block to the substrate along sliding direction. The surface roughness of $\mathrm{SiO}_{2}$ substrate was $0.18 \mathrm{~nm}$ in the simulation, comparable to 0.22 $\mathrm{nm}$ in the experiment.

In the simulated system, the top $2 \mathrm{~nm}$ of the tip and the bottom $1.5 \mathrm{~nm}$ of the substrate were held rigid. Adjacent to the rigid layer, thermostatted layers were created and held at $300 \mathrm{~K}$ using the Langevin thermostat. The remaining atoms were kept free. Periodic boundary conditions were applied in the $x$ - and $y$ - directions. The charge-optimized many-body (COMB) potential [25] and the large-scale atomic/molecular massively parallel simulator (LAMMPS) [26] were 
used to carry out the MD simulations. The COMB potential contains manybody effects that allow for the breaking of existing bonds and the formation of new bonds and it has been successfully applied in the interfacial structure of an $\mathrm{Si} / \mathrm{SiO}_{2}$ interface [25]. The time step was $0.2 \mathrm{fs}$ in all simulations.
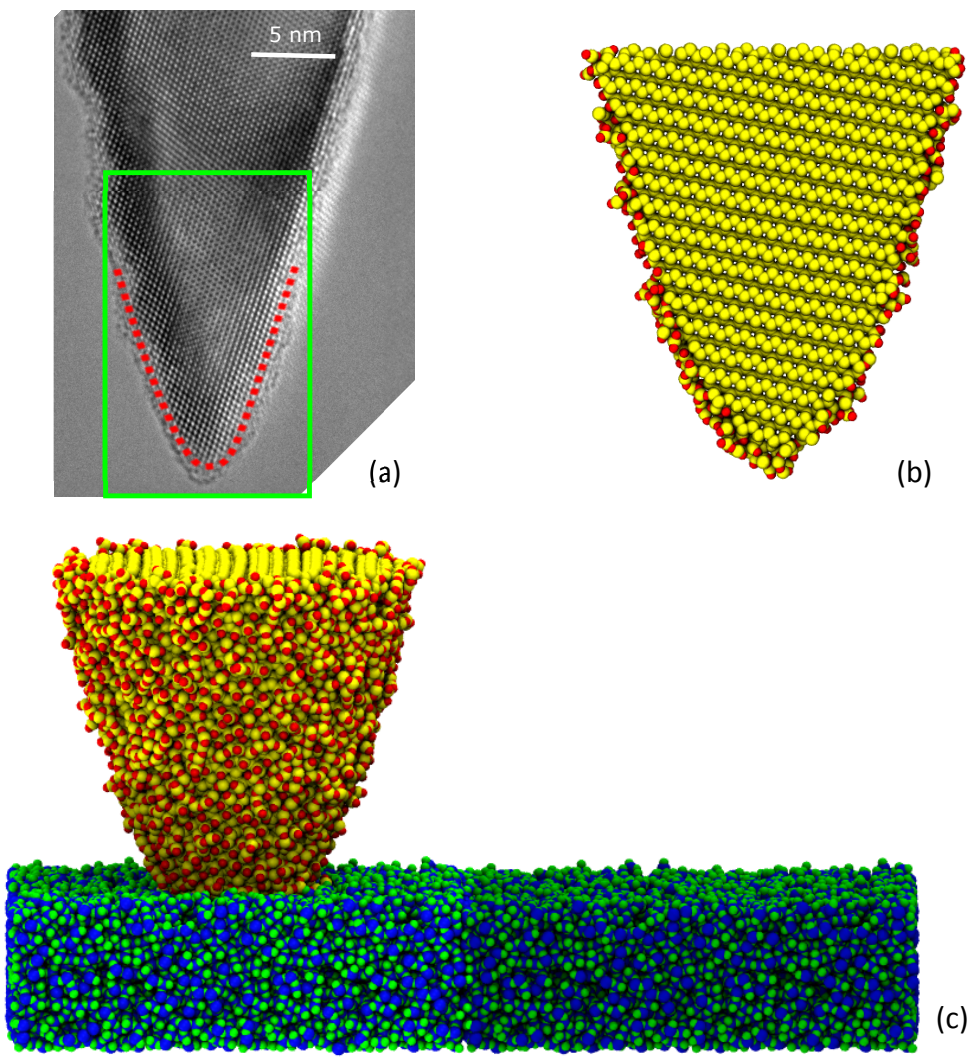

(c)

Figure 1: (a) TEM image of the silicon AFM tip before sliding, where the dashed line represents a parabola fit to the traced profile of the tip; (b) xz cross section of the model tip, which represents the Si ( $1-10)$ plane; (c) Snapshot of the simulation setup. The brightness change of the substrate indicates that the substrate can be extended along its sliding direction. Silicon atoms in the tip are shown in yellow, oxygen atoms in the tip in red, silicon atoms in the substrate in blue, and oxygen atoms in the substrate in green.

Indentation simulations were performed to partially validate the model. A load of $5 \mathrm{nN}$ was applied to the rigid layer of the tip in the $z\left[\begin{array}{lll}1 & 1 & 7\end{array}\right]$ direction. After the potential energy of the system was stable, which indicates that the 
system was at equilibrium, the tip was pulled away from the surface at a constant velocity of $20 \mathrm{~m} / \mathrm{s}$. The pull off force was identified as the minimum force during tip retraction. The pull off force measured in the simulation was $12.4 \pm$ $4.1 \mathrm{nN}$, which was reasonable, although somewhat larger than the $3.93 \pm 1.27$ $\mathrm{nN}$ measured in the experiment. The work of adhesion in the simulation was estimated to be $2 \pm 0.7 \mathrm{~J} / \mathrm{m}^{2}$ from Derjaguin-Muller-Toporov (DMT) contact model [27].

Sliding simulations were then performed to observe tip wear due to shear. A constant normal load of $100 \mathrm{nN}$ was applied on the rigid top layer of the tip and then the system was relaxed for $40 \mathrm{ps,}$ after which the potential energy was stable. Then, the tip was slid along either the $x$ - or the $y$ - direction at a constant speed of $10 \mathrm{~m} / \mathrm{s}$. Here, the $y\left[\begin{array}{lll}1 & -1 & 0\end{array}\right]$ direction was the same as the sliding direction in the experiment.

To quantify wear, the tip was pulled away from the surface at a constant velocity of $20 \mathrm{~m} / \mathrm{s}$ after sliding a given distance. Any silicon atom that was removed from the tip was considered to be worn. Then the worn volume was estimated by multiplying the number of worn atoms by the volume per atom, which was taken to be $0.02 \mathrm{~nm}^{3}$ for a silicon atom in a perfect lattice. The number of silicon atoms changing from crystalline to amorphous after sliding a given distance was calculated using OVITO [28], in which the diamond lattice of silicon is identified using an extended common neighbor analysis [29]. By multiplying the volume per atom, this number can be converted to the tip volume that changed from crystalline to amorphous due to sliding.

\section{Results and discussion}

We characterized the tip wear volume as a function of sliding distance for two different sliding directions as shown in Figure 2. It can be seen that the tip wear volume in the simulation increased with sliding distance in both sliding directions and that the sliding direction had little effect on wear volume, as expected for an amorphous substrate. However, after sliding $40 \mathrm{~nm}$, the tip wear 
volume in the experiment was larger (approximately four times) than that in the simulation. We then calculated the wear rate as a function of sliding distance along the $x$ - direction. From the inset in Figure 2, it can be seen that the wear rate decreased with sliding distance, consistent with the observed in previous AFM measurements of nanoscale wear [9]. It also can be seen that the wear rate is nearly constant by the end of the test, indicating an approach to steady state. The wear rate in the simulation was estimated to be $0.28 \times 10^{-2} \mathrm{~nm}^{2} / \mathrm{nN}$ at a sliding distance of $40 \mathrm{~nm}$. In the experiment, at the same distance, the wear rate was estimated as $1.15 \times 10^{-2} \mathrm{~nm}^{2} / \mathrm{nN}$, larger than that in the simulation. One possible reason for this difference is the sliding speed, as discussed next.

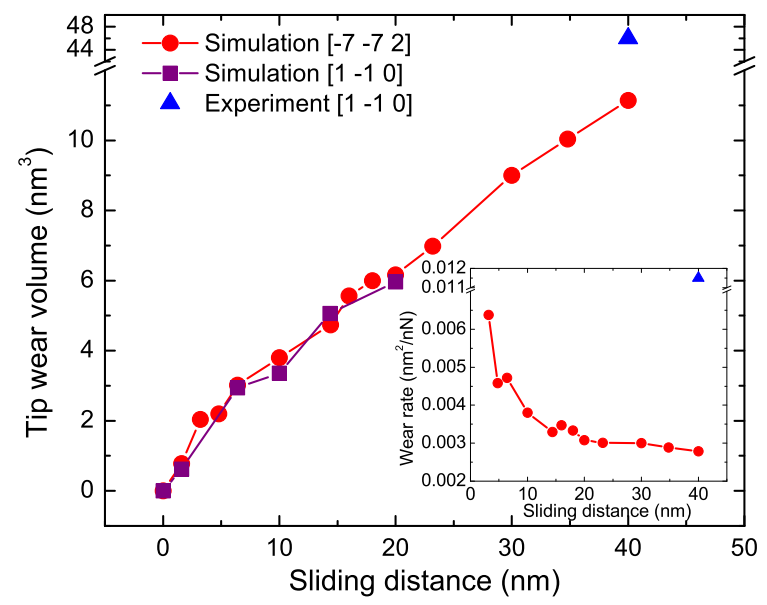

Figure 2: Tip wear volume as a function of sliding distance:red circles represent simulationcalculated wear volume for sliding direction [-7 -7 2], purple squares represent simulationcalculated wear volume for sliding direction $\left[\begin{array}{lll}1 & -1 & 0\end{array}\right]$, and the blue triangle represents experimentally measured wear volume for sliding direction $\left[\begin{array}{lll}1 & -1 & 0\end{array}\right]$. The inset shows the wear rate as a function of sliding distance for sliding direction[-7 - 72 2].

The sliding speed in the simulation was $10 \mathrm{~m} / \mathrm{s}$, which is orders of magnitude larger than the speed of $2.5 \mu \mathrm{m} / \mathrm{s}$ in the experiment. As suggested previously, nanoscale wear may occur through a process of atom-by-atom removal, which involves bond formation and bond breaking at the sliding interface [6]. Within the framework, a smaller sliding speed would provide more time for tip atoms 
and substrate atoms to form bonds and more bond breaking events are expected during sliding. This is supported by observations from a recent experimental study where an increase in sliding speed caused a decrease in the wear rate of nanocrystalline $\mathrm{Ni}[30]$. To investigate the effect of sliding speed on wear, we tested additional sliding speeds of $5 \mathrm{~m} / \mathrm{s}$ and $20 \mathrm{~m} / \mathrm{s}$ in the simulations. We did not observe any statistically significant difference between the wear rate calculated at these different speeds (less than $2 \%$ difference between wear rates at 5,10 and $20 \mathrm{~m} / \mathrm{s}$ ). However, the speed in the experiment was much slower than that in the simulation, which can be expected to be the most important factor to contribute to the difference in wear of materials.

We next analyze the amorphization process exhibited in the simulation. During tip indentation, we have observed that crystalline silicon atoms near the end of the tip became amorphous as shown in the inset (a) of Figure 3. It can be seen that the amorphous silicon atoms in the tip form a shape that may be related to the profile expected for the pressure distribution given by Hertz [31]. We then analyze the potential contribution of amorphization to sliding wear. First, evidence of this mechanism was seen in the fact that the worn tip material left on the substrate surface was amorphous; an example of the removal of amorphous material from the bottom of the tip is shown in the inset (b) of Figure 3. This observation is consistent with previous experimental studies which showed that the wear particles formed from crystalline diamond sliding on diamond were mainly amorphous carbon [18]. Second, we quantified this effect in the simulation as the volume of amorphous tip material. As shown in Figure 3, the amount of amorphous material (relative to the amount after indentation and before sliding) increased with sliding distance. Both of these observations suggest that amorphization is happening during sliding and that it may be part of the wear process.

In a previous study, an amorphization model was proposed to explain the time evolution of the thickness of amorphous carbon during diamond polishing [19]. In this model, crystalline atoms experience random collisions by amorphous atoms and then have a probability to become amorphous. Based on this model, 


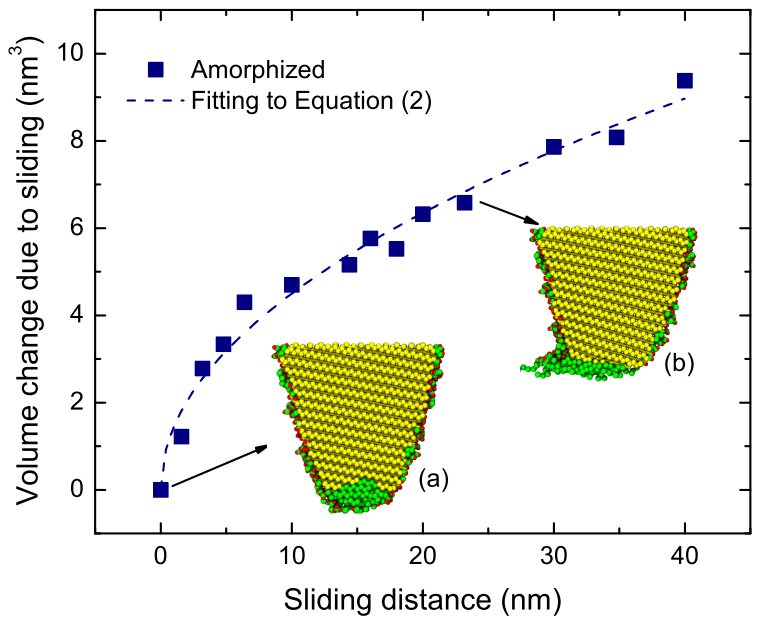

Figure 3: Amorphous volume change as a function of sliding distance at for the [-7 - 72$]$ sliding direction, where the navy dashed line represents the fitting of Equation 2 to the simulation data. The inset (a) shows the (1 -10$)$ plane of tip with amorphous atoms after indentation. The inset (b) shows the ( $\left.\begin{array}{lll}1 & -1 & 0\end{array}\right)$ plane of tip with amorphous atoms near the contact about to be removed due to wear. In this image, crystalline silicon atoms are shown in yellow, amorphous silicon atoms in green, and oxygen atoms in red.

the thickness of the amorphous material $h(t)$ can be expressed as

$$
h(t)=-\beta+\sqrt{\left(\beta+h\left(t_{0}\right)\right)^{2}+2\left(C_{1} P_{1}+C_{2} P_{2}\right) \alpha v_{0}\left(t-t_{0}\right)}
$$

where constants $\alpha$ and $\beta$ are dependent on the Herschel-Bulkley parameters; $t_{0}$ and $h\left(t_{0}\right)$ are the initial time and thickness of amorphous carbon, respectively; $C_{1}$ and $C_{2}$ are geometry constants for two sliding surfaces, $P_{1}$ and $P_{2}$ are the corresponding probabilities of a successful amorphization event, which are dependent on crystal direction.

To analyze amorphization in our simulation, we convert amorphous thickness $h(t)$ to amorphous volume $V$ by $V=A h(t)$, where we assume that the contact area $A$ changes little with time. In addition, $v_{0}\left(t-t_{0}\right)$ can be replaced by the sliding distance $L$. Thus, we can obtain that

$$
V=-\beta^{\prime}+\sqrt{\gamma+\alpha^{\prime} L}
$$

where $\beta^{\prime}, \gamma$ and $\alpha^{\prime}$ are constants, which are dependent on parameters $\alpha, \beta, t_{0}$, 
$h\left(t_{0}\right), C_{1}, P_{1}$ and $A$ in Equation 1. From Equation 2, it can be seen that the change in the amorphous volume is proportional to the square root of sliding distance. We then fit the data from MD to Equation 2. The fitting curve is shown in Figure 3 and the R-squared value of the fitting is 0.98 , indicating that the behavior predicted by the theory agrees well with simulation observations.

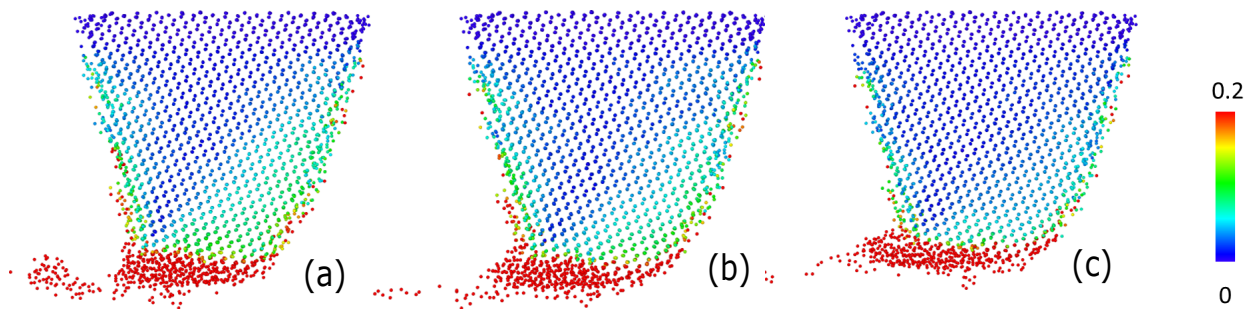

Figure 4: The (1 - $\left.\begin{array}{lll}1 & 0\end{array}\right)$ plane of silicon atoms in the tip after sliding (a) $6.4 \mathrm{~nm}$, (b) $14.4 \mathrm{~nm}$ and (c) $23.2 \mathrm{~nm}$. The color indicates local atomic shear strain.

The remaining question is why the amorphization rate decreases with sliding distance. To explore this, we analyzed the strain distribution within the tip. Strain is quantified using OVITO as the von Mises shear strain per atom [28, 32, 33], where the reference atomic configuration for the strain calculation is taken to be the configuration just before sliding begins in the simulation. The results at three representative moments in time are shown in Figure 4. Early in the sliding process, we observe large shear strain in the lower right region of the tip. However, as sliding continues, this strain is gradually released. A crystallineto-amorphous transition in silicon can be assisted by strain [34]. Therefore, the observation that strain is released during sliding can be used to explain the decreasing rate of amorphization.

To relate the amorphization to the wear, we plot amorphization rate and wear rate as functions of sliding distance as shown in Figure 5. Here, the amorphization rate was defined in a similar way as the definition of wear rate, i.e., the change of amorphous tip volume per sliding distance and applied load. It can be seen that both rates decreased with sliding distance and that the amorphization rate exhibits a similar trend to the wear rate, which suggests 


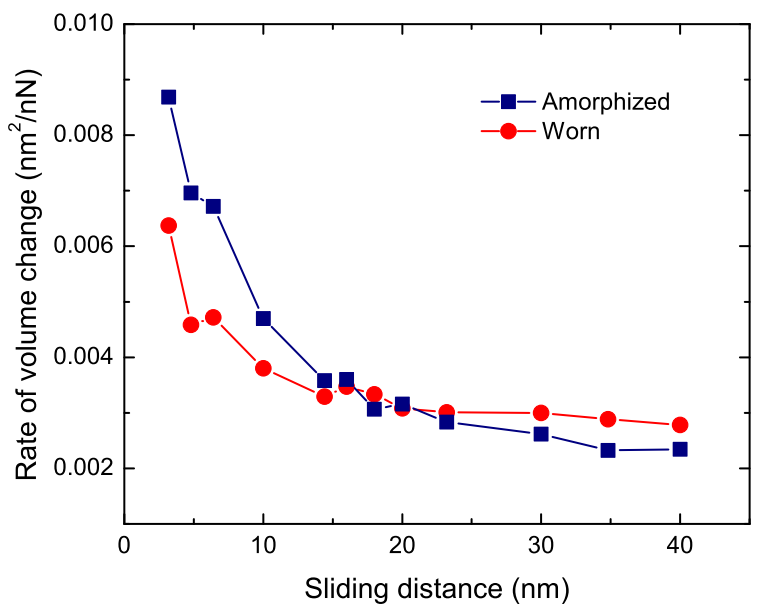

Figure 5: Amorphization and wear rate as functions of sliding distance.

that wear process may be assisted by amorphization. In addition, initially the amorphization rate is larger than wear rate, but after sliding, the trend is opposite with the wear rate being larger. This indicates that, during running-in, first tip atoms become amorphous and then are worn away. However, as the system approaches steady state, and both the amorphization and wear rates are nearly constant, the amorphization rate is smaller than wear rate. This implies that wear will eventually occur directly on the crystalline plane of tip, although this should be verified by further simulations and experiments. In general, these observations suggest that amorphization may be a dominant mechanism of wear during the early stages of running-in, but less so during steady-state sliding.

\section{Conclusions}

In summary, MD simulation was used to study nanoscale wear of a silicon AFM tip with a native oxide during the first $40 \mathrm{~nm}$ sliding against an amorphous silicon dioxide substrate. The tip wear volume was quantified as a function of sliding distance. We found that tip wear increases with sliding distance and the wear rate decreases with sliding distance. Based on the change of wear rate with sliding distance, the beginning of the wear transition from running-in to 
steady state was observed. The wear rate in the simulation was observed to be smaller than that in the experiment. The velocity in the experiment was much slower than that in the simulation, which can possibly contribute to the difference in wear of materials. The change of amorphous tip volume due to sliding was also quantified. The change of amorphous tip volume and sliding distance had a square-root relationship, which shows that the amorphization model can be applied to silicon crystal besides carbon. We also considered wear rate and amorphization rate as functions of sliding distance. Both rates decreased, which was explained qualitatively by a release of local strain within the tip. Further, the similar trends suggest that wear process was possibly assisted by amorphization of the material during running-in. In addition, in the initial sliding, the amorphization rate was larger than wear rate, but after

sliding, the wear rate became larger than amorphization rate, which suggests a possibility that wear will occur directly on the crystalline plane of tip at steady state. Thus, our study has shown the amorphization and wear trends during the initial sliding, which provides insight into the mechanisms of shear-induced silicon amorphization and nanoscale wear.

\section{Acknowledgements}

Work at the Molecular Foundry was supported by the Office of Science, Office of Basic Energy Sciences, of the U.S. Department of Energy under Contract No. DE-AC02-05CH11231. AM and XH acknowledge helpful discussions with TzuRay Shan related to the charge-optimized many-body potential.

\section{References}

[1] F. F. Abraham, I. P. Batra, S. Ciraci, Effect of tip profile on atomic-force microscope images: a model study, Phys. Rev. Lett. 60 (1988) 1314-1317.

[2] C. Odin, J. Aimé, Z. El Kaakour, T. Bouhacina, Tip's finite size effects on atomic force microscopy in the contact mode: simple geometrical consider- 
ations for rapid estimation of apex radius and tip angle based on the study of polystyrene latex balls, Surf. Sci. 317 (1994) 321-340.

[3] M. A. Lantz, B. Gotsmann, P. Jaroenapibal, T. D. Jacobs, S. D. O'Connor, K. Sridharan, R. W. Carpick, Wear-resistant nanoscale silicon carbide tips for scanning probe applications, Adv. Funct. Mater. 22 (2012) 1639-1645.

[4] J. Liu, D. Grierson, N. Moldovan, J. Notbohm, S. Li, P. Jaroenapibal, S. O'Connor, A. Sumant, N. Neelakantan, J. Carlisle, K. Turner, R. Carpick, Preventing nanoscale wear of atomic force microscopy tips through the use of monolithic ultrananocrystalline diamond probes, Small 6 (2010) 1140-1149.

[5] H. Edwards, R. McGlothlin, U. Elisa, Vertical metrology using scanningprobe microscopes: imaging distortions and measurement repeatability, J. Appl. Phys. 83 (1998) 3952-3971.

[6] T. D. Jacobs, R. W. Carpick, Nanoscale wear as a stress-assisted chemical reaction, Nat. Nanotechnol. (2013) 108-112.

[7] B. Gotsmann, M. A. Lantz, Atomistic wear in a single asperity sliding contact, Phys. Rev. Lett. (2008) 125501.

[8] A. Li, Y. Liu, I. Szlufarska, Effects of interfacial bonding on friction and wear at silica/silica interfaces, Tribol. Lett. 56 (2014) 481-490.

[9] H. Bhaskaran, B. Gotsmann, A. Sebastian, U. Drechsler, M. A. Lantz, M. Despont, P. Jaroenapibal, R. W. Carpick, Y. Chen, K. Sridharan, Ultralow nanoscale wear through atom-by-atom attrition in silicon-containing diamond-like carbon, Nat. Nanotechnol. 5 (2010) 181-185.

[10] R. Aghababaei, D. H. Warner, J.-F. Molinari, Critical length scale controls adhesive wear mechanisms, Nat. Commun. 7 (2016) 1-8.

[11] X. Hu, C. J. Tourek, Z. Ye, S. Sundararajan, A. Martini, Structural and chemical evolution of the near-apex region of an atomic force microscope tip subject to sliding, Tribol. Lett. 53 (2014) 181-187. 
[12] Y. Yang, L. Huang, Y. Shi, Adhesion suppresses atomic wear in singleasperity sliding, Wear 352 (2016) 31-41.

[13] L. Dai, V. Sorkin, Y. Zhang, Effect of surface chemistry on the mechanisms and governing laws of friction and wear, ACS Appl. Mater. \& Interfaces 8 (2016) 8765-8772.

[14] A. Yacoot, L. Koenders, Aspects of scanning force microscope probes and their effects on dimensional measurement, J. Phys. D Appl. Phys. 41 (2008) 103001.

[15] J. Furustig, I. Dobryden, A. Almqvist, N. Almqvist, R. Larsson, The measurement of wear using AFM and wear interpretation using a contact mechanics coupled wear model, Wear 350-351.

[16] Z.-D. Sha, V. Sorkin, P. S. Branicio, Q.-X. Pei, Y.-W. Zhang, D. J. Srolovitz, Large-scale molecular dynamics simulations of wear in diamondlike carbon at the nanoscale, Appl. Phys. Lett. 103 (2013) 073118.

[17] A. R. Konicek, D. Grierson, P. Gilbert, W. Sawyer, A. Sumant, R. W. Carpick, Origin of ultralow friction and wear in ultrananocrystalline diamond, Phys. Rev. Lett. 100 (2008) 235502.

[18] F. Van Bouwelen, A. Bleloch, J. Field, L. Brown, Wear by friction between diamonds studied by electron microscopical techniques, Diam. Relat. Mater. 5 (1996) 654-657.

[19] L. Pastewka, S. Moser, P. Gumbsch, M. Moseler, Anisotropic mechanical amorphization drives wear in diamond, Nat. Mater. 10 (2011) 34-38.

[20] D. Mondal, S. Das, R. Rao, M. Singh, Effect of SiC addition and runningin-wear on the sliding wear behaviour of $\mathrm{Al}-\mathrm{Zn}-\mathrm{Mg}$ aluminium alloy, Mater. Sci. Eng. A 402 (2005) 307-319.

[21] Y.-R. Jeng, Z.-W. Lin, S.-H. Shyu, Changes of surface topography during running-in process, J. Tribol. 126 (2004) 620-625. 
[22] L. Chen, S. H. Kim, X. Wang, L. Qian, Running-in process of $\mathrm{Si}-\mathrm{SiO}_{x} / \mathrm{SiO}_{2}$ pair at nanoscale-sharp drops in friction and wear rate during initial cycles, Friction 1 (2013) 81-91.

[23] W. Humphrey, A. Dalke, K. Schulten, VMD: visual molecular dynamics, J. Mol. Graphics 14 (1996) 33-38.

[24] E. R. Cruz-Chu, A. Aksimentiev, K. Schulten, Water-silica force field for simulating nanodevices, J. Phys. Chem. B 110 (2006) 21497-21508.

[25] T.-R. Shan, B. D. Devine, J. M. Hawkins, A. Asthagiri, S. R. Phillpot, S. B. Sinnott, et al., Second-generation charge-optimized many-body potential for $\mathrm{Si} / \mathrm{SiO}_{2}$ and amorphous silica, Phys. Rev. B 82 (2010) 235302.

[26] S. Plimpton, Fast parallel algorithms for short-range molecular dynamics, J. Comput. Phys. 117 (1995) 1-19.

[27] B. V. Derjaguin, V. M. Muller, Y. P. Toporov, Effect of contact deformations on the adhesion of particles, J. Colloid. Interf. Sci. 53 (1975) 314-326.

[28] A. Stukowski, Visualization and analysis of atomistic simulation data with OVITO-the Open Visualization Tool, Model. Simul. Mater. Sci. 18 (2009) 015012 .

[29] E. Maras, O. Trushin, A. Stukowski, T. Ala-Nissila, H. Jonsson, Global transition path search for dislocation formation in $\mathrm{Ge}$ on $\mathrm{Si}$ (001), Comput. Phys. Commun. 205 (2016) 13-21.

[30] M. Shafiei, A. Alpas, Effect of sliding speed on friction and wear behaviour of nanocrystalline nickel tested in an argon atmosphere, Wear 265 (2008) $429-438$.

[31] K. L. Johnson, Contact mechanics, Cambridge University Press, 1987.

[32] F. Shimizu, S. Ogata, J. Li, Theory of shear banding in metallic glasses and molecular dynamics calculations, Mater. Trans. 48 (2007) 2923-2927. 
[33] A. Cao, Y. Cheng, E. Ma, Structural processes that initiate shear localization in metallic glass, Acta Mater. 57 (2009) 5146-5155.

[34] Y.-C. Wang, W. Zhang, L.-Y. Wang, Z. Zhuang, E. Ma, J. Li, Z.-W. Shan, In situ TEM study of deformation-induced crystalline-to-amorphous transition in silicon, NPG Asia Mater. 8 (2016) e291. 